\title{
CONSEQUÊNCIAS DO ISOLAMENTO SOCIAL NOS IDOSOS EM DOMICÍLIO EM TEMPOS DE PANDEMIA POR COVID-19
}

Eduardo Lopes Pereira; Universidade Federal do Pampa - UNIPAMPA; eduardoolopees@gmail.com Ana Karina Silva da Rocha Tanaka; Universidade Federal do Rio Grande do Sul - UFRGS; anakarinatanaka@gmail.com

Rosaura Soares Paczek; Universidade Federal do Rio Grande do Sul - UFRGS; rspaczek@gmail.com Letice Dalla Lana; Universidade Federal do Pampa - UNIPAMPA; letice.lana@gmail.com

\section{RESUM0}

Introdução: A COVID-19 requer medidas de combate à transmissão como higiene adequada das mãos e isolamento social, impactando no processo de envelhecimento saudável. Objetivos: Identificar consequências do isolamento social nos idosos. Método: Revisão da literatura sobre gerontologia, idosos, COVID-19 e isolamento social, com busca de artigos científicos publicados em 2020, bases de dados Scielo e Medline, foram utilizados 4 artigos. Resultados e discussão: A alta prevalência de óbito entre idosos pode desencadear medo constante, situações de estresse, ansiedade, solidão, tristeza e grande pressão psicológica. Alterações metabólicas ocorrem pelo isolamento social, atrofia muscular é considerada fisiológica no processo de envelhecimento reduzindo taxas de linfócitos na cavidade pulmonar, com redução da depuração das vias aéreas e reserva pulmonar. A restrição no domicílio potencializa os riscos para quedas, com a redução da massa e força muscular. As evidências científicas definem que a limitação imposta pelo isolamento social compromete o processo de envelhecimento saudável do idoso, nos aspectos social, fisiológico e psicológico. Deste modo, cabe aos profissionais da saúde propor medidas que reduzam as danos à saúde do idoso e que garantam o isolamento social durante a pandemia provocada pela COVID-19. Conclusão: Evidências demonstram que os idosos tiveram um destaque de atenção na pandemia, por ser um dos grupos de risco, com necessidade de instituir medidas de promoção e prevenção à saúde, os quais ampliam as redes de atenção à saúde da pessoa idosa incentivando as boas práticas como exercícios em casa ou atividades de memória.

Palavras-chave: "Idoso"; "Isolamento social”; "Pandemia."

\section{REFERÊNCIAS}

COSTA, Felipe de Almeida et al. COVID-19: seus impactos clínicos e psicológicos na população idosa. Brazilian Journal Of Development, [S.L.], v. 6, n. 7, p. 49811-4982, 2020. Brazilian Journal of Development. http://dx.doi. org/10.34117/bjdv6n7-580.

HAMMERSCHMIDT, Karina Silveira de Almeida; SANTANA, Rosimere Ferreira. SAÚDE DO IDOSO EM TEMPOS DE PANDEMIA COVID-19. Revista Cogitare Enfermagem, [S.L.], v. 25, n. 3, p. 1-10, 2020. Revista Cogitare Enfermagem. http:// dx.doi.org/10.5380/ce.v25i0.72849.

NASCIMENTO JÚNIOR, Francisco Edimar do; TATMATSU, Daniely Ildegardes Brito; FREITAS, Rayanne Gabrielle Torquato de. ANSIEDADE EM IDOSOS EM TEMPOS DE ISOLAMENTO SOCIAL NO BRASIL (COVID-19). Revista Brasileira de Análise do Comportamento, [S.L.], v. 16, n. 1, p. 50-56, 2020. Revista Brasileira de Análise do Comportamento. http://dx.doi.org/10.18542/rebac.v16i1.9097.

VELHO, Fábio Daniel; HERÉDIA, Vania Beatriz Merlotti. Quarantined senior citizens and the impact of technology on their life. Revista Rosa dos Ventos - Turismo e Hospitalidade, [S.L.], v. 12, p. 1-14, 12 jul. 2020. Universidade Caixias do Sul. http://dx.doi.org/10.18226/21789061.v12i3a10. 